\title{
Sarc-F and muscle function in community dwelling adults with aged care service needs: Baseline and post-training relationship
}

\author{
Justin W L Keogh ${ }^{\text {Corresp., 1, 2, 3, } 4 \text {, Tim Henwood }}{ }^{1,5,6}$, Paul A Gardiner ${ }^{7,8}$, Anthony G Tuckett ${ }^{9,10}$, Sharon Hetherington ${ }^{11}$, \\ Kevin Rouse ${ }^{11}$, Paul Swinton ${ }^{12}$

Background: This study sought to better understand the psychometric properties of the SARC-F, by examining the baseline and training-related relationships between the five SARC-F items and objective measures of muscle function. Each of the five items of the SARC-F are scored from 0-2, with total score of four or more indicative of likely sarcopenia.

Methods: This manuscript describes a sub-study of a larger step-wedge, randomised controlled 24-week progressive resistance and balance training (PRBT) program trial for Australian community dwelling older adults accessing government supported aged care. Muscle function was assessed using handgrip strength, isometric knee extension, 5-time repeated chair stand and walking speed over $4 \mathrm{~m}$. Associations within and between SARC-F categories and muscle function were assessed using multiple correspondence analysis (MCA) and multinomial regression, respectively. Results: Significant associations were identified at baseline between SARC-F total score and measures of lower-body muscle function ( $r=-0.62$ to $0.57 ; p \leq 0.002$ ) in 245 older adults. MCA analysis indicated the first three dimensions of the SARC-F data explained $48.5 \%$ of the cumulative variance. The initial dimension represented overall sarcopenia diagnosis, Dimension 2 the ability to displace the body vertically, and Dimension 3 walking ability and falls status. The majority of the 168 older adults who completed the PRBT program reported no change in their SARC-F diagnosis or individual item scores (56.5-79.2\%). However, significant associations were obtained between training-related changes in SARC-F total and item scores and changes in walking speed and chair stand test performance ( $r=-0.30$ to $0.33 ; p<0.001$ and relative risk ratio $=0.40-2.24 ; p<0.05$, respectively). MCA analysis of the change score data indicated that the first two dimensions explained $32.2 \%$ of the cumulative variance, with these

Peer) reviewing PDF | (2019:04:37069:1:1:NEW 11 Oct 2019) 
dimensions representing whether a change occurred and the direction of change, respectively. Discussion: The results advance our comprehension of the psychometric properties on the SARC-F, particularly its potential use in assessing changes in muscle function. Older adults' perception of their baseline and training-related changes in their function, as self-reported by the SARC-F, closely matched objectively measured muscle function tests. This is important as there may be a lack of concordance between self-reported and clinician-measured assessments of older adults' muscle function. However, the SARC-F has a relative lack of sensitivity to detecting training-related changes, even over a period of 24 weeks. Conclusions: Results of this study may provide clinicians and researchers a greater understanding of how they may use the SARC-F and its potential limitations. Future studies may wish to further examine the SARC-F's sensitivity of change, perhaps by adding a few additional items or an additional category of performance to each item. 
$1 \quad$ Sarc-F and muscle function in community dwelling adults with aged care service needs: Baseline and

2 the Sunshine Coast, Sunshine Coast, Australia

\section{${ }^{5}$ Southern Cross Care SA and NT, Adelaide, Australia}

${ }^{6}$ School of Human Movement and Nutritional Science, University of Queensland, Brisbane, Australia

${ }^{7}$ Faculty of Medicine, University of Queensland, Brisbane, Australia

${ }^{8}$ Mater Research Institute, University of Queensland, Brisbane, Australia

${ }^{9}$ University of Queensland, School of Nursing, Midwifery and Social Work, Brisbane, Australia

${ }^{10}$ College of Nursing, Yonsei University, Seoul, Korea

${ }^{11}$ The Chermside Senior Citizens Centre, Burnie Brae, Australia

${ }^{12}$ School of Health Sciences, Robert Gordon University, Aberdeen, Scotland 
20 Corresponding Author:

21 Justin Keogh

22 Faculty of Health Sciences and Medicine, Bond University, QLD 4229, Australia.

23 Email address: jkeogh@bond.edu.au

24

25

26 


\section{Abstract}

Background: This study sought to better understand the psychometric properties of the SARC-F, by examining the baseline and training-related relationships between the five SARC-F items and objective measures of muscle function. Each of the five items of the SARC-F are scored from 0-2, with total score of four or more indicative of likely sarcopenia.

Methods: This manuscript describes a sub-study of a larger step-wedge, randomised controlled 24-week progressive resistance and balance training (PRBT) program trial for Australian community dwelling older adults accessing government supported aged care. Muscle function was assessed using handgrip strength, isometric knee extension, 5-time repeated chair stand and walking speed over $4 \mathrm{~m}$.

Associations within and between SARC-F categories and muscle function were assessed using multiple correspondence analysis (MCA) and multinomial regression, respectively.

Results: Significant associations were identified at baseline between SARC-F total score and measures of lower-body muscle function $(r=-0.62$ to $0.57 ; p \leq 0.002)$ in 245 older adults. MCA analysis indicated the first three dimensions of the SARC-F data explained $48.5 \%$ of the cumulative variance. The initial dimension represented overall sarcopenia diagnosis, Dimension 2 the ability to displace the body vertically, and Dimension 3 walking ability and falls status. The majority of the 168 older adults who completed the PRBT program reported no change in their SARC-F diagnosis or individual item scores (56.5-79.2\%). However, significant associations were obtained between training-related changes in SARC- $F$ total and item scores and changes in walking speed and chair stand test performance $(r=-0.30$ to $0.33 ; p<0.001$ and relative risk ratio $=0.40-2.24 ; p<0.05$, respectively). MCA analysis of the change score data indicated that the first two dimensions explained $32.2 \%$ of the cumulative variance, with these dimensions representing whether a change occurred and the direction of change, respectively. 
49 Discussion: The results advance our comprehension of the psychometric properties on the SARC-F,

50 particularly its potential use in assessing changes in muscle function. Older adults' perception of their

51 baseline and training-related changes in their function, as self-reported by the SARC-F, closely matched

52 objectively measured muscle function tests. This is important as there may be a lack of concordance

53 between self-reported and clinician-measured assessments of older adults' muscle function. However,

54 the SARC-F has a relative lack of sensitivity to detecting training-related changes, even over a period of

5524 weeks.

56 Conclusions: Results of this study may provide clinicians and researchers a greater understanding of

57 how they may use the SARC-F and its potential limitations. Future studies may wish to further examine

58 the SARC-F's sensitivity of change, perhaps by adding a few additional items or an additional category of

59 performance to each item.

60 Key words: aged care; exercise; physical performance; sarcopenia; screening.

61

62

63 
64

65

66

67

68

69

70

71

72

\section{Introduction}

The SARC-F is a quick self-report sarcopenia screening tool involving five questions (items) assessing an older individual's muscular strength, ability to walk, rise from a chair and climb stairs as well as fall status (Malmstrom \& Morley 2013). It is endorsed for use in clinical practice and research by the European Working Group Sarcopenia in Older People (EWGSOP2) consensus (Cruz-Jentoft et al. 2019).

Previous research indicates that the SARC-F can predict quality of life, physical limitation, hospitalisation and mortality over periods up to 4 years post-assessment in older adults (Fougère \& Morley 2018; Woo et al. 2014; Wu et al. 2016). The SARC-F has reasonable validity and/or reliability when used with older adults across numerous countries and languages (Bahat et al. 2018; Cao et al. 2014; Ida et al. 2017; Malmstrom et al. 2015; Rodriguez-Rejon et al. 2018). The SARC-F typically demonstrates moderate to good specificity and negative predictive values, but poorer sensitivity and positive predictive values (Barbosa-Silva et al. 2016; Ida et al. 2017; Woo et al. 2014). Such results indicate that the SARC-F may be appropriate at correctly classifying older adults who are not sarcopenic; however, lower levels of sensitivity and positive predictive values indicate it is less able to accurately identify sarcopenic individuals. It is perhaps this poor level of sensitivity that has led the Australian and New Zealand Society for Sarcopenia and Frailty Research (ANZSSFR) to recommend additional research before the SARC-F be recommended for use in clinical practice (Zanker et al. 2019).

In addition to its somewhat questionable sensitivity, there appear to be two main gaps within the literature that may impact the SARC-F's potential use in clinical practice and research. The first gap reflects the relative lack of research that has examined the relationship between total SARC-F scores, individual SARC-F item scores and a range of objective muscle function tests, including those advocated by the EWGSOP2 such as handgrip strength, knee extension strength, chair stand performance and walking speed. The second gap reflects the lack of research that has examined the potential for the 
87 SARC-F to accurately quantify changes in sarcopenic diagnosis, muscle function and/or other important

88 health outcomes with proven interventions such as exercise and/or nutritional support. Exercise

89 (particularly progressive resistance training) and nutritional support (e.g. protein supplementation) are

being advocated as potential options to reduce the prevalence and severity of reduced physical

91

function/physical disability and sarcopenia in older adults (Cruz-Jentoft 2013; Denison et al. 2015).

While researchers investigating age-related disability can use a variety of criterion methods to assess

muscle function, clinicians working with patients are unlikely to have such time and tools available to

them. Therefore, if the SARC-F can detect clinically significant changes in muscle function as a result of

exercise and/or nutritional interventions, a variety of medical and allied health researchers/clinicians

may have a feasible tool to quantify the effects of these interventions.

the changes in SARC-F data observed in a step wedge randomised control intervention and their

may inform our interpretation of the findings relevant to the primary aim were to use a cross-sectional

research approach to: (1) examine how the SARC-F scale at baseline were related to objective measures

of muscle function; and (2) explore structure between SARC-F scale items at baseline.

\section{Materials and Methods}

105

106

107

108

109

\section{Study design}

The data reported in this manuscript describe the results of a sub-study of a single-blind larger trial, where the methods are fully described in a trial protocol (Keogh. et al. 2017) and within two papers describing different aspects of the overall trial's results (Hetherington et al. 2018; Hetherington et al. in press). Briefly, community-dwelling older Australians in receipt of government-funded aged care 
110 support were recruited to participate in an intervention comprising progressive resistance and balance

111 training (PRBT). In addition to holding an Australian government funded aged care package, the

112 participants were required to be: (1) over 65 years of age; (2) community-dwelling; (3) mobile with or

113 without an aid; (4) able to follow instructions and commit to the study period; and (5) with no recent

114 history (last six months) of resistance training. The exclusion criteria were: (1) requiring more than one

115 person to assist with transfers, standing and/or mobilising; (2) medications and/or diseases with

116 contraindications for exercise; (3) terminal illness or receiving palliative care; (4) an imminent move to

117 residential care; (5) cognitive decline and/or dementia that causes difficult and/or unpredictable

118 behaviours; and (6) inability to obtain a doctor's consent to participate.

All participants were recruited from the membership database of a large north-Brisbane

120 (Queensland, Australia) community and senior citizens centre that offered, among a suite of other

121 services, aged care support. On behalf of the CEO of the community and senior citizens centre, the

122 research manager sent a letter of invitation to this project to each of its members. A total of 388

123 individuals replied that they were interested in participating, with 349 identified as eligible by telephone

124 interview. These 349 eligible individuals were sent a study pack containing the participant information

125 sheet, the consent form, health history questionnaire and balance questionnaire and requested to

126 attend the exercise clinic for baseline assessment. Of these, 104 withdrew from the study prior to

127 baseline assessment. The exercise clinic in which the intervention was delivered was also used to

128 perform the baseline assessments. Following the baseline assessment, the 245 participants were

129 randomised by the research manager to exercise (PRBT) or wait list control (CON) at a ratio of 1:2 using

130 block randomisation through a sealed envelope selection method. A modified stepped-wedge

131 randomisation process was used to ensure all participants were provided an opportunity to participate

132 in the exercise intervention if they were interested. The sample of 245 participants was close to the

133 maximum projected 300 older adults who could be recruited to the study and trained in a safe and 
134 effective manner (Keogh. et al. 2017). The stepped-wedge design included four delivery phases of

135 participant entry (between September 2015 and August 2017) that resulted in eight exercise and control

136 clusters. As we aimed to include up to 300 participants in this trial by trial completion at the end of

137 2017, and working within the constraints of gym size and availability of accredited exercise physiologists,

138 the cluster sizes of the exercise and control groups exhibited some minor variation (Hetherington et al.

139 2018; Hetherington et al. in press).

Ethics approval was obtained from the University of Queensland Human Research Ethic

141 Committee (Approval number \#2015000879) and the study was registered with the Australian New

142 Zealand Clinical Trials Registry (ACTRN12615001153505). All participants provided written informed

143 consent prior to participation in this study.

\section{Intervention}

145 The duration of the exercise intervention was 24 weeks. Consistent with previous safe and successful

146 PBRT trials involving older adults with mobility limitations and complex health care requirements as well

147 as current recommendations based on meta-analyses, participants were asked to perform twice-weekly

148 PRBT (Fien et al. 2016; Fisher et al. 2017; Grgic et al. 2018). The resistance training exercises were

149 performed in a traditional manner (i.e. with heavy loads at relatively slow velocities), in an attempt to

150 maximise the muscular strength and to some extent the hypertrophy adaptations (Reid et al. 2015;

151 Schoenfeld et al. 2017; Stec et al. 2017). The balance training exercises included a mixture of static and

152 dynamic balance tasks as recommended to improve walking speed and to reduce the risk of falls and

153 other adverse age-related effects associated with low walking speed and/or low physical activity levels

154 (Abellan van Kan et al. 2009; Granacher et al. 2011).

155 Exercise classes involved a light five-minute warm-up, 45 minutes of PRBT exercises followed by

156 a 5-minute cool down incorporating stretches. All exercise classes involved no more than 10 participants 
157 and were performed under the supervision of accredited exercise physiologists who were experienced

158 working with older clients with chronic disease. Resistance exercises were performed on air-pressure 159 driven, computer-integrated machines proven effective for use among older adults (HUR Australia Pty

160 Ltd, Birkdale, QLD, Australia (Hewitt et al. 2018). The program is overviewed in detail in Supplementary

161 Figure 1, and a more detailed overview of the program can be found in the MUAD trial protocol (Keogh.

162 et al. 2017). If participants experienced pain or discomfort when performing any exercise, the exercise

163 was modified or removed from the participant's program.

Wait list CON participants were requested to continue their regular activities for the initial 24-

165

166

167

168

170

171

172

173

174

175

176

177 178 179 weeks period. This was evaluated through the completion of a daily dairy. Diaries were completed by all participants during their time in the study, and collected data on sleep integrity, exercise and physical activity engagement, falls and GP, hospital and clinician presentations. These data are outside the scope of this manuscript, but no change in physical activity patterns during the CON phase were observed. To aid retention, the CON group received monthly education seminars covering a range of health and wellness topics. Following the 24-weeks phase, the CON group were given the opportunity to participate in the 24-weeks exercise phase.

\section{Data collection}

A detailed description of the outcome measures collected in the larger trial is provided in the trial protocol (Keogh. et al. 2017). In summary, data collection occurred at baseline (week 0) and postintervention (week 25) for all participants enrolled in this study, with the assessors blinded to the participants' allocation. Participants completed the SARC-F self-report questionaire (Malmstrom \& Morley 2013). The SARC-F questionnaire comprises five individual items (components) that are each scored from 0 to 2 . The first four items assess the older individual with regards to slowness in walking, assistance walking, chair rise and stair climbing ability graded on a scale that represents no (0), some (1)

PeerJ reviewing PDF | (2019:04:37069:1:1:NEW 11 Oct 2019) 
180 or an inability (2) to perform a given task, respectively. The final question of the SARC-F assesses their

181 falls history in the last year: no falls (0), 1-3 falls (1) and 4 or more falls (2). Total SARC-F scores of four or 182 more are suggestive of sarcopenia (Malmstrom \& Morley 2013).

In accordance with the recommendation of the EWGSOP (Cruz-Jentoft 2013), muscle function

184

185

186

was assessed by isometric handgrip strength, Isometric knee extension strength, 5-time repeated chair stand and walking speed. While a short description of these methods is provided below, more details can be found in the trial protocol (Keogh. et al. 2017). Upper body strength was assessed in the handgrip exercise using an isometric Jamar dynamometer (Sammons Preston Roylan, Bolingbrook, IL). Three trials of the dominant hand were performed, with the best trials retained for analysis (Schaap et al. 2016). Isometric leg extension strength was assessed with a 0-500 kg strain gauge HUR Performance Recorder (HUR Labs Oy, Tampere, FI) that was positioned on the leg extension machine. With the knee locked at $45^{\circ}$, the participant was requested to push against the machine with maximum force.

Participants performed two trials of the isometric knee extension with the best result retained. Physical performance was assessed by the participant's habitual walking speed over $4 \mathrm{~m}$ and 5-time repeated chair stands (Guralnik et al. 1994; Studenski et al. 2011). As per the established protocol, one trial of the 5-time chairs stand was completed and two trials of the walking speed test were performed, with the best trial retained. If a participant was unable to complete five repetitions of the chair stand test within the maximum time frame of one minute (60 s), they were given a score of $61 \mathrm{~s}$.

Aspects of this wider dataset were used in the present sub-study to describe the demographic characteristics of the sample. These include a variety of anthropometric (body mass, body mass index $B M I)$, general health (medications and morbidities), function (Activities of Balance Confidence $-A B C$ ), mood (Geriatric Depression Scale and Geriatric Anxiety Index) and quality of life (EQ-5D-3L - EuorQoL 5D 3L) measures that have been validated for use in older populations (Herdman et al. 2011; Kurlowicz 
203 1999; Mak et al. 2012; Moore et al. 2011; Pachana et al. 2007). A full description of these methods is

204 provided elsewhere (Keogh. et al. 2017).

205

206

\section{Statistical Analysis}

207

208

209

210

211

212

213

214

215

216

217

218

219

220

221

222

223

224

Data collected from the SARC-F scale at baseline were used to: (1) explore relationships with objective measures of muscle function; and (2) explore structure between scale items. Linear and multinomial regression models were used to investigate relationships between objective measures of muscle function and SARC-F total score and SARC-F Item scores, respectively. Exploration of underlying structure in SARC-F responses was completed using multiple correspondence analysis (MCA). MCA is a factor analysis technique that is similar to other descriptive methods such as cluster analysis and principal component analysis but operates on categorical data. MCA combines quantitative and graphical methods to assess structure in measurement tools such as the SARC-F that comprise categorical outcomes, and is becoming more widespread in studies involving older adults (Costa et al. 2013; Marcucci et al. 2017). MCA was selected to describe patterns of responses between the five individual SARC-F items to better understand use of the tool within the specific population. Briefly, the technique assesses deviation from independence between items and identifies relationships in a lower dimensional space that can be visualised. Permissible responses (zero, one or two) for each of the five items comprising the SARC-F questionnaire were plotted with a marker in the lower dimensional space with the first dimension representing the largest deviation from independence and the second dimension (orthogonal to the first) representing the next largest deviation from independence. Interpretation of each dimension was based on relative positions of category markers, with markers closest to the axis and furthest from the origin identified as most important in describing a dimension. 
227 models, pre to post SARC-F items were coded as no-change, positive-change or negative-change.

228 Multinomial models also controlled for baseline response, with $p$-values calculated for regression

229 coefficients using Wald tests. Finally, structural changes in SARC-F items across the intervention were

230 explored with MCA. All statistical analyses were conducted in the R programming language ( $R$

231 Development Core Team, Vienna, Austria) with MCA performed with the FactoMineR package (Husson

232 et al. 2010) and multinomial logistic regression models estimated with the nnet package (Venables \&

233 Ripley 2002).

234

\section{Results}

236 A summary of the participants who were randomised to this trial and those who completed the exercise

237 program is provided in Figure 1. The baseline characteristics of the participants are summarised in Table

238 1. There were no significant differences between those who completed the exercise intervention

239 compared to those who only attended the initial assessment.

241 Please insert Figure 1 and Table 1 about here

244 isometric knee extension strength, walking speed and chair-stand test performance $(p \leq 0.002)$. Values

245 presented in Table 2 provide estimates of the relative risk ratio for a one standard deviation increase in performance variables responding negatively to a SARC-F item compared with a positive response. 
248 Please insert Table 2 about here

249

Eigenvalues obtained from MCA with baseline SARC-F data indicated the first 3 dimensions

251 (48.5\% cumulative variance) warranted exploration. Results are presented in Figures 2 and 3 with

252 sarcopenia diagnosis (diagnosed from SARC-F) plotted as a supplementary qualitative variable.

Dimension 1 represents overall sarcopenia diagnosis, with negative values for Dimension 1 representing non-sarcopenic individuals and positive values representing sarcopenic individuals. The ability to displace the body vertically (rising from a chair and climbing stairs) appeared to best explain deviation and falls status items were best represented in Dimension 3 (see Figure 3).

Please insert Figure 2 and 3 about here SARC-F items (56.5-78.0\%) or their sarcopenia diagnosis based on their total SARC-F score (79.2\%).

Please insert Table 3 about here 
269 isometric knee extension strength $(p=0.452)$ or handgrip strength $(p=0.878)$. Similar results were

270 obtained for multinomial logistic regression models (Table 4), where the training-related changes in

271 walking speed chair-stand test performance were significantly associated with changes in SARC-F items

272 response.

273

274 Please insert Table 4 about here

275

276

Eigenvalues obtained from MCA with the training-related change in SARC-F items indicated that

277 the first two dimensions (32.2\% cumulative variance) warranted exploration. Results of the MCA

278 analysis are presented in Figure 4 with the training-related change in the SARC-F sarcopenic diagnosis plotted as a supplementary qualitative variable. Dimension 1 is represented by change in SARC-F items,

with no change to the left of the origin and change (positive and negative) to the right, with Dimension 2

281 represented by the direction of the change in SARC-F items. Figure 4 indicates a consistency in response with, for example, those experiencing positive change in one item more likely to experience a positive change in another item.

Please insert Figure 4 about here

Discussion

This study demonstrated stronger relationships between the SARC-F total and physical performance measures of chair-stand test performance and walking speed $(r=-0.30-0.33$, respectively) than knee 
290

291

292

extension or handgrip strength. These relationships were particularly strong for the chair stand test, whereby a one standard deviation reduction in performance resulted in an $\sim 2-5$ times increased risk of a negative change in all five SARC-F item scores.

The results of the MCA on the baseline data identified three MCA dimensions that accounted for $48.5 \%$ of the cumulative variance of the SARC-F scores, -with the first and most influential dimension separating positive (no difficulty) and negative responses (a lot of difficulty) in completing a task. In contrast, the second dimension was associated with moving the body vertically during chair stands and stair climbing, indicating this ability is influential in explaining variation in item response. Similarly, the third dimension was primarily associated with ambulation, based on differentiating responses related to the ability to walk independently and risk of falls. These last two dimensions of physical function (i.e. ability to successfully move the body vertically or horizontally) have been extensively studied in a variety of older adult cohorts as these tasks appear pivotal in maintaining function, independence and overall health and well-being with increasing age (Abellan van Kan et al. 2009; Bean et al. 2007; Howe \& Skelton 2011; Keogh et al. 2015). Collectively, the results of the present study and the literature suggest that the SARC-F may be a useful self-report tool from which older adults can describe their current level of physical function and falls risk.

Examination of the training-related responses indicated significant improvements in the two lower body strength-related outcomes (i.e. the chair stand test and isometric knee extension strength), with trends observed for increased walking speed when compared to the tendency for decline in the control group. Such results appear typical for progressive resistance training interventions, whereby all older adults will significantly increase their muscle strength but exhibit more variable and smaller responses in walking speed and other mobility tasks (Barbalho et al. 2017; Churchward-Venne et al. 2015; Fien et al. 2016). 

performance were observed after 24 weeks of PRBT, the majority of individuals reported no change in individual SARC-F item scores (56.5-78.0\%) or their SARC-F diagnosis (79.2\%). This lack of sensitivity to change in the SARC-F scores is consistent with the wider sarcopenia literature, whereby previous studies observed no significant change in sarcopenia (assessed with either the SARC-F or EWGSOP), even though significant improvements in muscle function were observed (Cruz-Jentoft et al. 2014; Hassan et al. 2016). Another possible explanation for the lack of change in SARC-F items or sarcopenia diagnosis within the current study may reflect the fact that only $34.0 \%$ of the sample who completed the exercise program were initially diagnosed by the SARC-F as sarcopenic. Collectively, the overall lack of change in the SARC-F items and total score for the PRBT group reinforce some of the limitations regarding its psychometric properties (Barbosa-Silva et al. 2016; Ida et al. 2017; Woo et al. 2014). Linear regression models did however demonstrate that changes in the SARC-F total score were significantly associated with objective changes in walking speed and chair stand test performance $\left(r^{2}=0.08-0.10\right)$. The strength of these relationships was greatest for chair stand performance, whereby a one standard deviation change (increase or decrease) in objectively measured chair stand performance resulted in close to twice the probability (RR 1.98-2.24) of a one point change in the SARC-F strength, walking, chair rise and stair climbing item scores. Similar results were obtained with multinomial regression models used to account for the categorical nature of individual SARC-F items, where associations between the change in item response were generally associated with changes to in walking speed and chair-stand test performance. Further, MCA results demonstrated that participants who improved performance in one SARC-F item were more likely to also report improved performance in other item(s). Such an outcome supports the importance of lower body muscle strength 
Our results therefore suggest that older adults may be able to identify clinically significant

changes in their muscle function via the SARC-F that correspond to those identified through objective assessments performed by health professionals. This correspondence between health professional derived objective and older adult self-reported subjective muscle function measures is an important finding that many previous studies have been unable to replicate (Cyarto et al. 2008; Liu-Ambrose et al. 2004; van Delden et al. 2013). Perhaps the inconsistency between our results and the literature reflects the SARC-F's brevity and simplicity compared to the other tools used in these studies (i.e. Activities of Balance Confidence, Community Balance and Mobility Scale, Stroke Impact Scale - Hand and Motor Activity Log) (Cyarto et al. 2008; Liu-Ambrose et al. 2004; van Delden et al. 2013). adults may help track their progress and positively contribute to their longer term adherence to the intervention, as older individuals who self-report meaningful benefits from exercise are more likely to continue exercising than those who did not perceive such benefits (Fisken et al. 2015; Hamlin et al. 2016). Such studies may also look to compare the original SARC-F to a modified SARC-F, as the use of a five item, three-point scale to quantify muscle function and falls risk may not be sufficiently sensitive, with this relative lack of sensitivity likely contributing to possible ceiling and/or floor effects. Currently, one such modified SARC-F has been examined, whereby the addition of the measurement of calf girth demonstrated significantly greater sensitivity in the diagnosis of sarcopenia when compared to the SARC-F alone (Bahat et al. 2018). Future research may examine how the inclusion of an additional category per SARC-F item may improve the psychometric properties of the SARC-F. This could be achieved by separating a score of 2 (currently described as "A lot or unable") into two scores (2 - "A lot

357 of trouble" or 3 - "Unable to perform"). Such a modification may make it easier to demonstrate meaning that the total score would be out of 15 instead of 10 . 
were: (1) Its focus on an understudied large older adult population at high risk of entry into residential aged care due to their reduced muscle function and (2) our innovative examination of the SARC-F's relationship before and after long-term PRBT training. The results of this study have implications to the community and aged care sector, whereby an easy to use screening tool for declining muscle function and geriatric syndromes such as sarcopenia will assist a variety of healthcare professionals better identify older adults who need assistance to retain/improve their physical independence and function if they wish to continue living in the community. In contrast, two primary limitations were: (1) that a pragmatic stepped-wedge randomised control trial design was utilised whereby some of the results may restricted to a moderate velocity format.

\section{Conclusions}

Investigation of the underlying structure of the SARC-F screening tool and its relationship to objective measures of muscle function revealed a number of relevant findings for clinicians. A range of moderate and strong associations were observed with both total SARC-F score and individual SARC-F items, to objective measures of muscle function at baseline and with change across 24 weeks of PRBT. Overall, these findings provide additional insight into the psychometric properties of the SARC-F and provide

378 some preliminary support for how the SARC-F may be used to identify meaningful changes in older 379 adults' muscle function as a result of performing interventions such as resistance training. 
382 We acknowledge the contribution of the exercise physiologists and support staff at Healthy Connections

383 Exercise Clinic to the success of this project. We also acknowledge our project partners HUR Australia

384 Pty Ltd, St Vincent's Health Australia, and Burnie Brae. Without Burnie Brae's commitment to health and

385 wellbeing, this project would not have been possible. 


\section{References}

Abellan van Kan G, Rolland Y, Andrieu S, Bauer J, Beauchet O, Bonnefoy M, Cesari M, Donini LM, Gillette Guyonnet S, Inzitari M, Nourhashemi F, Onder G, Ritz P, Salva A, Visser M, and Vellas B. 2009. Gait speed at usual pace as a predictor of adverse outcomes in community-dwelling older people an International Academy on Nutrition and Aging (IANA) Task Force. Journal of Nutrition, Health and Aging 13:881-889.

Bahat G, Yilmaz O, Kiliç C, Oren MM, and Karan MA. 2018. Performance of SARC-F in regard to sarcopenia definitions, muscle mass and functional measures. Journal of Nutrition, Health and Aging. 10.1007/s12603-018-1067-8

Barbalho MSM, Gentil P, Izquierdo M, Fisher J, Steele J, and Raiol RA. 2017. There are no no-responders to low or high resistance training volumes among older women. Experimental Gerontology 99:18-26. 10.1016/j.exger.2017.09.003

Barbosa-Silva TG, Menezes AM, Bielemann RM, Malmstrom TK, and Gonzalez MC. 2016. Enhancing SARC-F: improving sarcopenia screening in the clinical practice. Journal of the American Medical Directors Association 17:1136-1141. 10.1016/j.jamda.2016.08.004

Bean JF, Kiely DK, LaRose S, Alian J, and Frontera WR. 2007. Is stair climb power a clinically relevant measure of leg power impairments in at-risk older adults? Archives of Physical Medicine and Rehabilitation 88:604-609.

Cao L, Chen S, Zou C, Ding X, Gao L, Liao Z, Liu G, Malmstrom TK, Morley JE, Flaherty JH, An Y, and Dong B. 2014. A pilot study of the SARC-F scale on screening sarcopenia and physical disability in the Chinese older people. Journal of Nutrition, Health and Aging 18:277-283. 10.1007/s12603-0130410-3

Churchward-Venne TA, Tieland M, Verdijk LB, Leenders M, Dirks ML, de Groot LC, and van Loon L. 2015. There are no nonresponders to resistance-type exercise training in older men and women. Journal of the American Medical Directors Association 16:400-411. 10.1016/j.jamda.2015.01.071

Costa PS, Santos NC, Cunha P, Cotter J, and Sousa N. 2013. The use of multiple correspondence analysis to explore associations between categories of qualitative variables in healthy ageing. Journal of Aging Research 2013:302163-302163. 10.1155/2013/302163

Cruz-Jentoft AJ. 2013. Perspective: protein and exercise for frailty and sarcopenia: still learning. Journal of the American Medical Directors Association 14:69-71. 10.1016/j.jamda.2012.09.024

Cruz-Jentoft AJ, Bahat G, Bauer J, Boirie Y, Bruyère O, Cederholm T, Cooper C, Landi F, Rolland Y, Sayer AA, Schneider SM, Sieber CC, Topinkova E, Vandewoude M, Visser M, Zamboni M, Writing Group for the European Working Group on Sarcopenia in Older People 2 (EWGSOP2), and the Extended Group for EWGSOP2. 2019. Sarcopenia: revised European consensus on definition and diagnosis. Age and Ageing 48:16-31. 10.1093/ageing/afy169

Cruz-Jentoft AJ, Landi F, Schneider SM, Zuniga C, Arai H, Boirie Y, Chen LK, Fielding RA, Martin FC, Michel JP, Sieber C, Stout JR, Studenski SA, Vellas B, Woo J, Zamboni M, and Cederholm T. 2014. Prevalence of and interventions for sarcopenia in ageing adults: a systematic review. Report of the International Sarcopenia Initiative (EWGSOP and IWGS). Age and Ageing 43:748-759. 10.1093/ageing/afu115

Cyarto EV, Brown WJ, Marshall AL, and Trost SG. 2008. Comparative effects of home- and group-based exercise on balance confidence and balance ability in older adults: cluster randomized trial. Gerontology 54:272-280. 10.1159/000155653

Denison HJ, Cooper C, Sayer AA, and Robinson SM. 2015. Prevention and optimal management of sarcopenia: a review of combined exercise and nutrition interventions to improve muscle outcomes in older people. Clinical Interventions in Aging 10:859-869. 
432

433

434

435

436

437

438

439

440

441

442

443

444

445

446

447

448

449

450

451

452

453

454

455

456

457

458

459

460

461

462

463

464

465

466

467

468

469

470

471

472

473

474

475

476

477

478

Fien S, Henwood T, Climstein M, and Keogh JWL. 2016. Feasibility and benefits of group-based exercise in residential aged care adults: a pilot study for the GrACE programme. PeerJ 4:e2018. 10.7717/peerj.2018

Fisher JP, Steele J, Gentil P, Giessing J, and Westcott WL. 2017. A minimal dose approach to resistance training for the older adult; the prophylactic for aging. Experimental Gerontology 99:80-86. 10.1016/j.exger.2017.09.012

Fisken A, Keogh JWL, Waters D, and Hing W. 2015. Perceived benefits, motives, and barriers to aquabased exercise among older adults with and without osteoarthritis. Journal of Applied Gerontology 34:377-396. 10.1177/0733464812463431

Fougère B, and Morley JE. 2018. Rapid screening for frailty and sarcopenia in daily clinical practice. Journal of Nutrition, Health and Aging. 10.1007/s12603-018-1057-x

Granacher U, Muehlbauer T, Gollhofer A, Kressig RW, and Zahner L. 2011. An intergenerational approach in the promotion of balance and strength for fall prevention: A mini-review. Gerontology 57:304-315.

Grgic J, Schoenfeld BJ, Davies TB, Lazinica B, Krieger JW, and Pedisic Z. 2018. Effect of resistance training frequency on gains in muscular strength: A systematic review and meta-analysis. Sports Medicine 48:1207-1220. 10.1007/s40279-018-0872-x

Guralnik JM, Simonsick EM, Ferrucci L, Glynn RJ, Berkman LF, Blazer DG, Scherr PA, and Wallace RB. 1994. A short physical performance battery assessing lower extremity function: association with self-reported disability and prediction of mortality and nursing home admission. J Gerontol 49:M85-94.

Hamlin MJ, Yule E, Elliot CA, Stoner L, and Kathiravel Y. 2016. Long-term effectiveness of the New Zealand Green Prescription primary health care exercise initiative. Public Health 140:102-108. 10.1016/j.puhe.2016.07.014

Hassan BH, Hewitt J, Keogh JW, Bermeo S, Duque G, and Henwood TR. 2016. Impact of resistance training on sarcopenia in nursing care facilities: a pilot study. Geriatric Nursing 37:116-121. 10.1016/j.gerinurse.2015.11.001

Herdman M, Gudex C, Lloyd A, Janssen M, Kind P, Parkin D, Bonsel G, and Badia X. 2011. Development and preliminary testing of the new five-level version of EQ-5D (EQ-5D-5L). Qual Life Res 20:17271736. 10.1007/s11136-011-9903-x

Hetherington S, Henwood T, Swinton P, Keogh J, Gardiner P, Tuckett A, and Rouse K. 2018. Engineering improved balance confidence in older adults with complex health care needs: learning from the Muscling Up Against Disability Study. Archives of Physical Medicine and Rehabilitation 99:15251532. 10.1016/j.apmr.2018.03.004

Hetherington S, Swinton P, Henwood T, Keogh J, Gardiner P, Tuckett A, Rouse K, and Comans T. in press. Progressive resistance plus balance training for older Australians receiving in-home care services: Cost-effectiveness analyses alongside the Muscling Up Against Disability steppedwedge randomized control trial. Journal of Physical Activity and Aging.

Hewitt J, Goodall S, Clemson L, Henwood T, and Refshauge K. 2018. Progressive resistance and balance training for falls prevention in long-term residential aged care: a cluster randomized trial of the Sunbeam Program. Journal of the American Medical Directors Association 19:361-369. 10.1016/j.jamda.2017.12.014

Howe TE, and Skelton DA. 2011. Consensus on core outcome measures of function are needed to progress our knowledge of 'best practice' exercise components for older people. Age and Ageing 40:532-533. 10.1093/ageing/afr082

Husson F, Josse J, Le S, and Mazet J. 2010. FactoMineR: Multivariate exploratory data analysis and data mining with R. Available at http://cran.r-project.org/package=FactoMineR. 
479

480

481

482

483

484

485

486

487

488

489

490

491

492

493

494

495

496

497

498

499

500

501

502

503

504

505

506

507

508

509

510

511

512

513

514

515

516

517

518

519

520

521

522

523

524

525

Ida S, Murata K, Nakadachi D, Ishihara Y, Imataka K, Uchida A, Monguchi K, Kaneko R, Fujiwara R, and Takahashi H. 2017. Development of a Japanese version of the SARC-F for diabetic patients: an examination of reliability and validity. Aging Clinical and Experimental Research 9:935-942. 10.1007/s40520-016-0668-5

Keogh JW, Senior H, Beller EM, and Henwood T. 2015. Prevalence and risk factors for low habitual walking speed in nursing home residents: an observational study. Archives of Physical Medicine and Rehabilitation 96:1993-1999. 10.1016/j.apmr.2015.06.021

Keogh. JWL, Henwood T, Gardiner P, Tuckett A, Hodgkinson B, and Rouse K. 2017. Examining evidence based resistance plus balance training in community-dwelling older adults with complex health care needs: trial protocol for the Muscling Up Against Disability project. Archives of Gerontology and Geriatrics 68:97-105.

Kurlowicz L. 1999. The Geriatric Depression Scale (GDS). Geriatric Nursing 20:212-213.

Liu-Ambrose T, Khan KM, Eng JJ, Lord SR, and McKay HA. 2004. Balance confidence improves with resistance or agility training. Increase is not correlated with objective changes in fall risk and physical abilities. Gerontology 50:373-382.

Mak MK, Pang MY, and Mok V. 2012. Gait difficulty, postural instability, and muscle weakness are associated with fear of falling in people with Parkinson's disease. Parkinson's disease 2012:901721. 10.1155/2012/901721

Malmstrom TK, Miller DK, Simonsick EM, Ferrucci L, and Morley JE. 2015. SARC-F: a symptom score to predict persons with sarcopenia at risk for poor functional outcomes. Journal of Cachexia, Sarcopenia and Muscle 7:28-36. 10.1002/jcsm.12048

Malmstrom TK, and Morley JE. 2013. SARC-F: a simple questionnaire to rapidly diagnose sarcopenia. Journal of the American Medical Directors Association 14:531-532.

Marcucci M, Franchi C, Nobili A, Mannucci PM, and Ardoino I. 2017. Defining aging phenotypes and related outcomes: Clues to recognize frailty in hospitalized older patients. Journals of Gerontology Series A: Biological Sciences and Medical Sciences 72:395-402. 10.1093/gerona/glw188

Moore DS, Ellis R, Kosma M, Fabre JM, McCarter KS, and Wood RH. 2011. Comparison of the validity of four fall-related psychological measures in a community-based falls risk screening. Research quarterly for exercise and sport 82:545-554.

Pachana NA, Byrne GJ, Siddle H, Koloski N, Harley E, and Arnold E. 2007. Development and validation of the Geriatric Anxiety Inventory. Int Psychogeriatr 19:103-114. 10.1017/S1041610206003504

Reid KF, Martin KI, Doros G, Clark DJ, Hau C, Patten C, Phillips EM, Frontera WR, and Fielding RA. 2015. Comparative effects of light or heavy resistance power training for improving lower extremity power and physical performance in mobility-limited older adults. Journals of Gerontology Series A: Biological Sciences and Medical Sciences 70:374-380. 10.1093/gerona/glu156

Rodriguez-Rejon Al, Artacho R, Puerta A, Zuñiga A, and Ruiz-Lopez MD. 2018. Diagnosis of sarcopenia in long-term care homes for the elderly: the sensitivity and specificity of two simplified algorithms with respect to the EWGSOP consensus. Journal of Nutrition, Health and Aging 22:796-801. 10.1007/s12603-018-1004-x

Schaap LA, Fox B, Henwood T, Bruyère O, Reginster JY, Beaudart C, Buckinx F, Roberts H, Cooper C, Cherubini A, dell'Aquilla G, Maggio M, and Volpato S. 2016. Grip strength measurement: Towards a standardized approach in sarcopenia research and practice. European Geriatric Medicine 7:247-255. 10.1016/j.eurger.2015.11.012

Schoenfeld BJ, Grgic J, Ogborn D, and Krieger JW. 2017. Strength and hypertrophy adaptations between low- vs. High-load resistance training: A systematic review and meta-analysis. Journal of Strength and Conditioning Research 31:3508-3523. 10.1519/jsc.0000000000002200

Peer) reviewing PDF | (2019:04:37069:1:1:NEW 11 Oct 2019) 
526
Stec MJ, Thalacker-Mercer A, Mayhew DL, Kelly NA, Tuggle SC, Merritt EK, Brown CJ, Windham ST, Dell'Italia L, Bickel CS, Roberts BM, Vaughn KM, Isakova-Donahue I, Many GM, and Bamman MM. 2017. Randomized, four-arm, dose-response clinical trial to optimize resistance exercise training for older adults with age-related muscle atrophy. Experimental Gerontology 99:98-109. 10.1016/j.exger.2017.09.018

Studenski S, Perera S, Patel K, Rosano C, Faulkner K, Inzitari M, Brach J, Chandler J, Cawthon P, Connor EB, Nevitt M, Visser M, Kritchevsky S, Badinelli S, Harris T, Newman AB, Cauley J, Ferrucci L, and Guralnik J. 2011. Gait speed and survival in older adults. JAMA 305:50-58. 10.1001/jama.2010.1923

van Delden AEQ, Peper CE, Beek PJ, and Kwakkel G. 2013. Match and mismatch between objective and subjective improvements in upper limb function after stroke. Disability and Rehabilitation 35:1961-1967. 10.3109/09638288.2013.768303

Venables W, N. , and Ripley BD. 2002. Modern applied statistics with S-PLUS. New York: Springer.

Woo J, Leung J, and Morley JE. 2014. Validating the SARC-F: a suitable community screening tool for sarcopenia? Journal of the American Medical Directors Association 15:630-634. 10.1016/j.jamda.2014.04.021

Wu T-Y, Liaw C-K, Chen F-C, Kuo K-L, Chie W-C, and Yang R-S. 2016. Sarcopenia screened with SARC-F questionnaire is associated with quality of life and 4-year mortality. Journal of the American Medical Directors Association 17:1129-1135.

Zanker J, Scott D, Reijnierse EM, Brennan-Olsen SL, Daly RM, Girgis CM, Grossmann M, Hayes A, Henwood T, Hirani V, Inderjeeth CA, Iuliano S, Keogh JWL, Lewis JR, Maier AB, Pasco JA, Phu S, Sanders KM, Sim M, Visvanathan R, Waters DL, Yu SCY, and Duque G. 2019. Establishing an Operational Definition of Sarcopenia in Australia and New Zealand: Delphi Method Based Consensus Statement. The Journal of Nutrition, Health \& Aging 23:105-110. 10.1007/s12603018-1113-6 
Figure 1

Participant flow diagram.

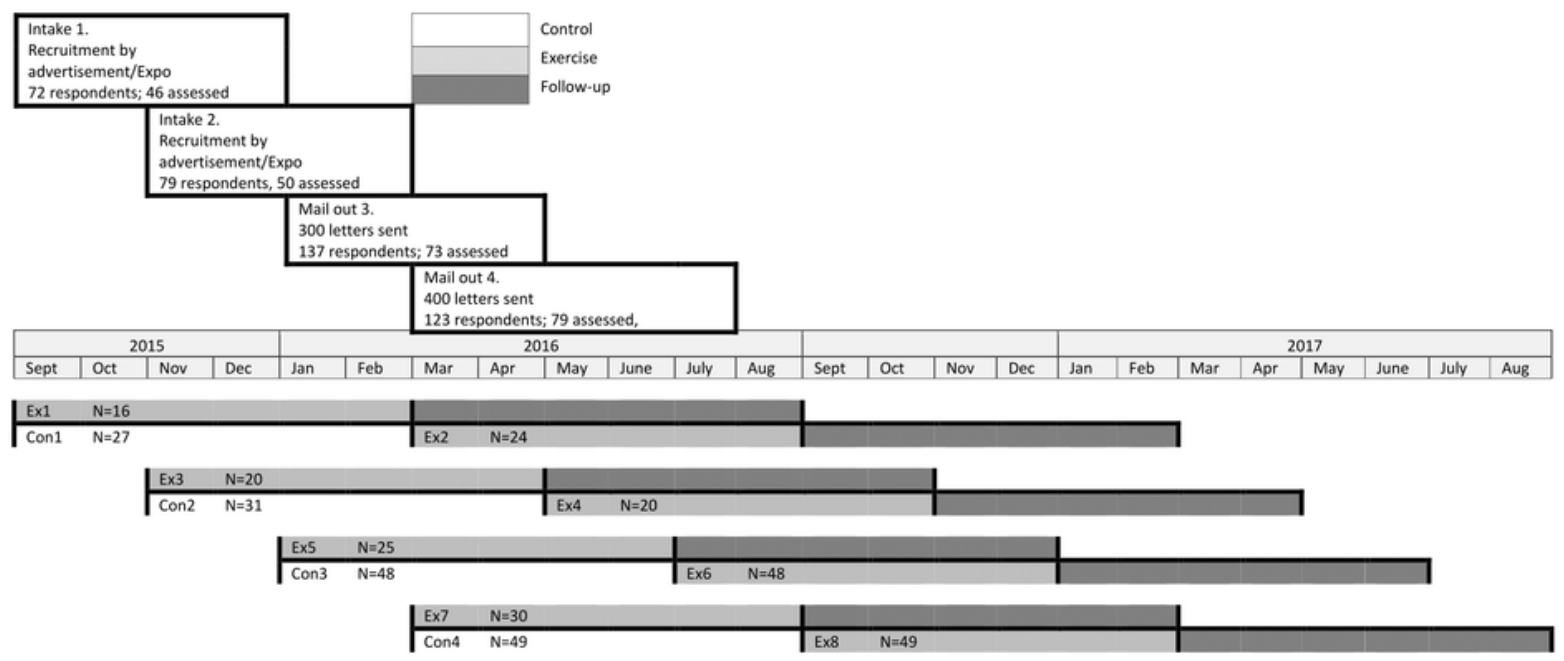


Figure 2

Graphical representation of Multiple Correspondence Analysis (MCA) of baseline SARC-F responses (Dimension 1 vs Dimension2).

Factor map identifying most influential categories for dimensions presented along with projected supplementary vectors (Sarcopenic and non-sarcopenic).

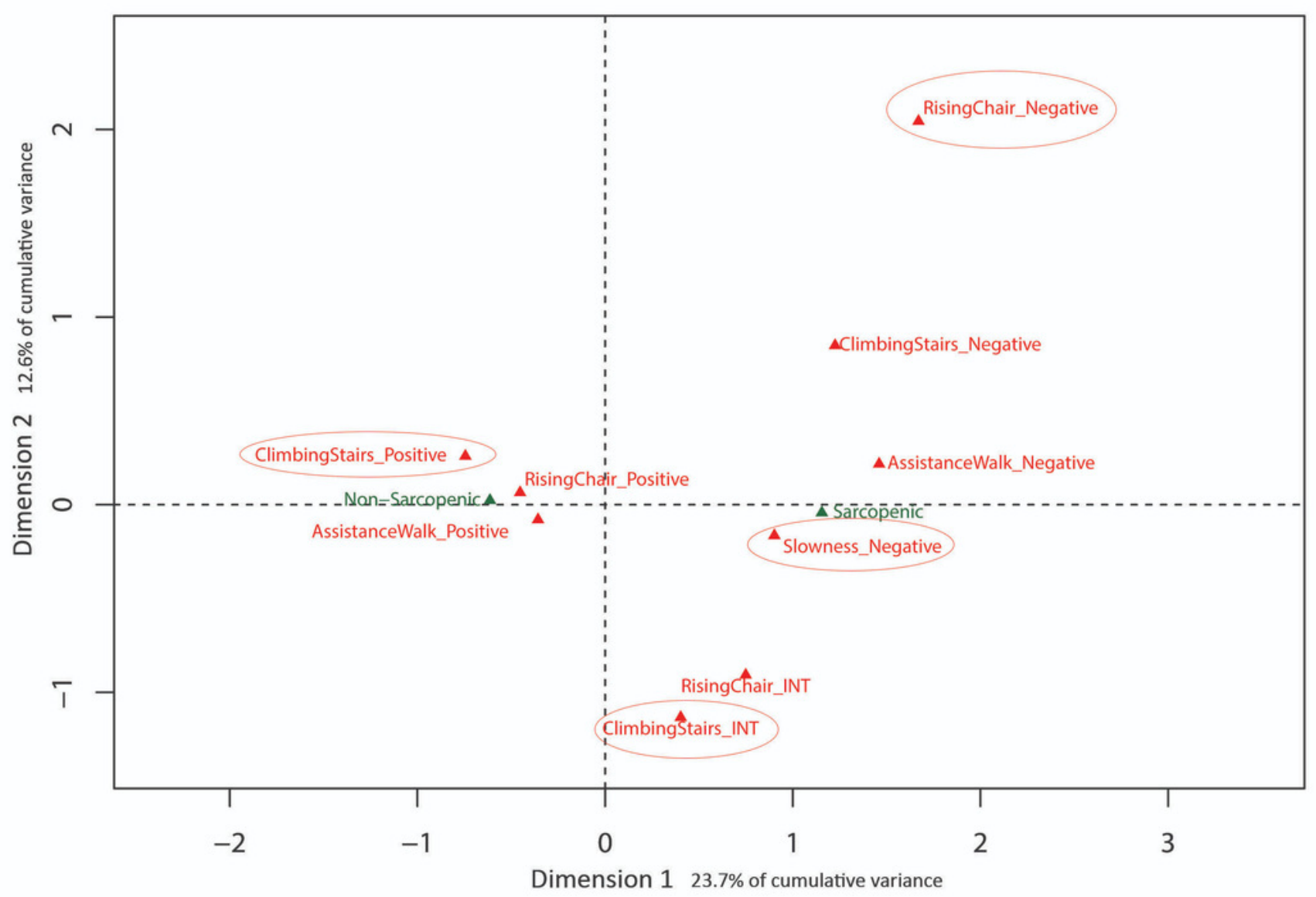


Figure 3

Graphical representation of Multiple Correspondence Analysis (MCA) of baseline SARC-F responses (Dimension 1 vs Dimension 3).

Factor map identifying most influential categories for dimensions presented along with projected supplementary vectors (Sarcopenic and non-sarcopenic).

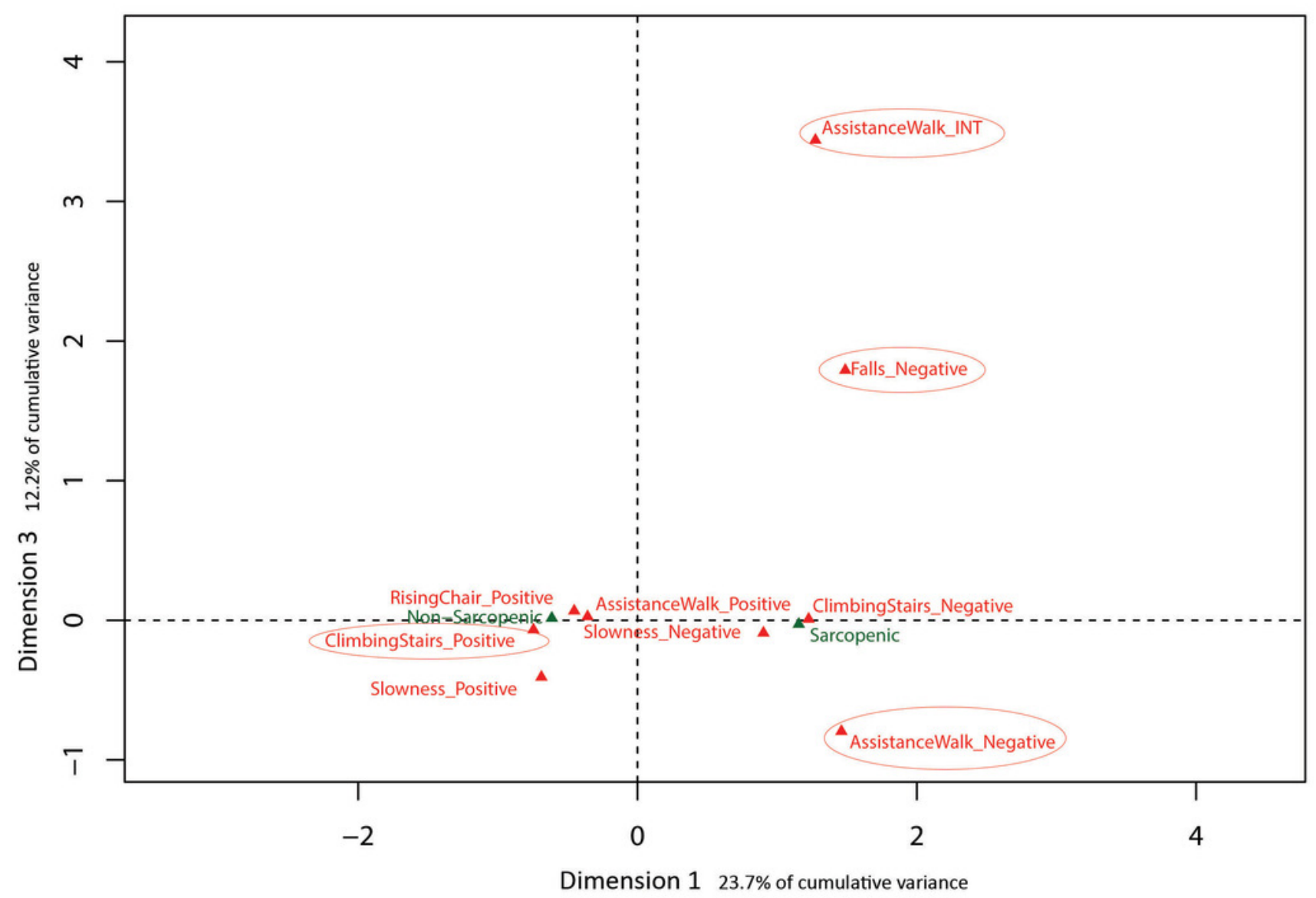


Figure 4

Graphical representation of Multiple Correspondence Analysis (MCA) of change in SARC$\mathrm{F}$ responses across intervention (Dimension 1 vs Dimension 2).

Factor map identifying most influential categories for dimensions presented along with projected supplementary vectors (Sarcopenic and non-sarcopenic).

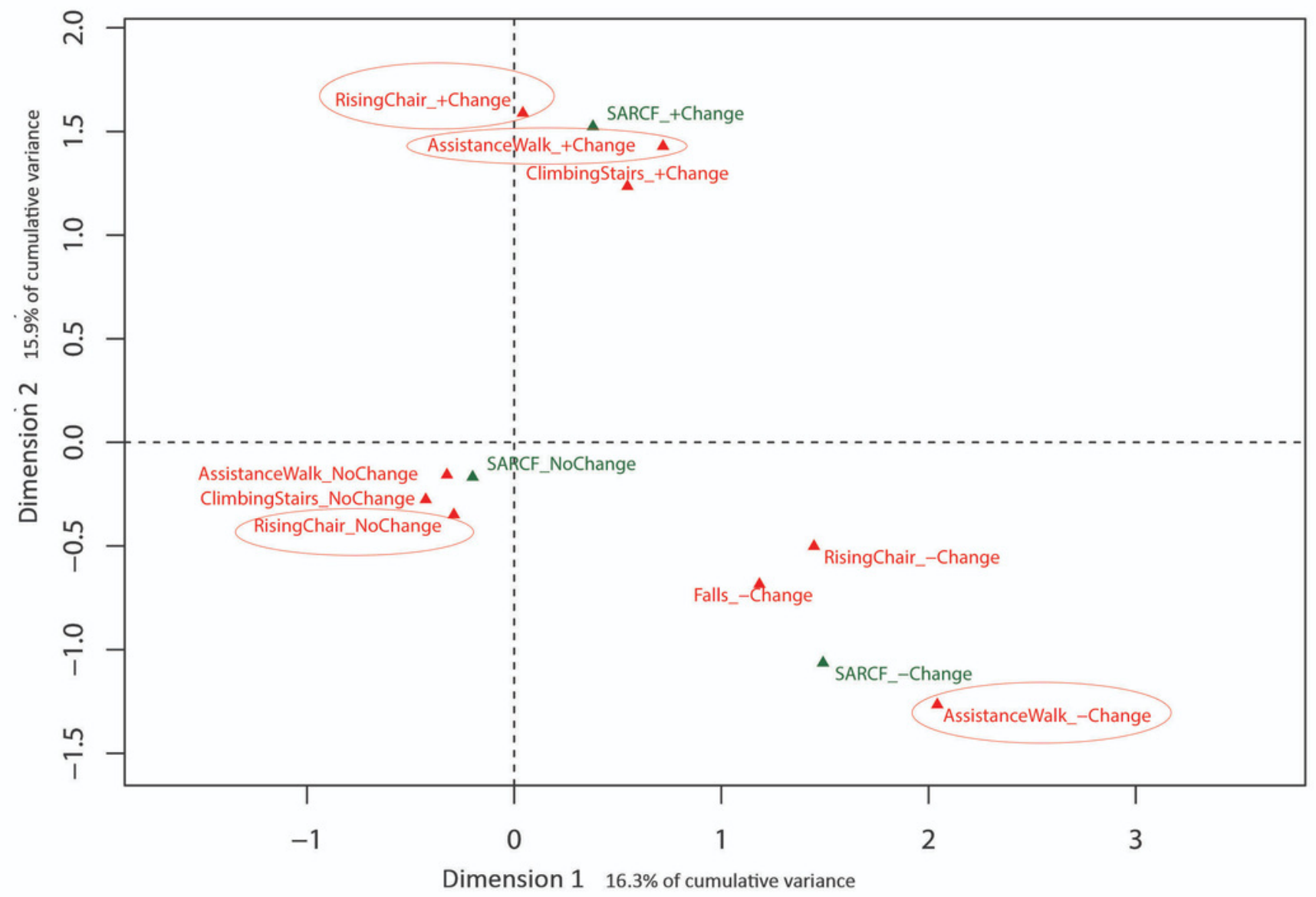




\section{Table $\mathbf{1}$ (on next page)}

Baseline characteristics of participants.

All continuous data are presented as mean \pm standard deviation. All categorical data are presented as the absolute number followed by the percentage. ABC: Activity-specific Balance Confidence questionnaire. SPPB: Short Physical performance Battery. GDS: Geriatric Depression Scale. GAl: Geriatric Anxiety Index. EQ-5D-3L: EuorQoL 5D 3L questionnaire. 
1 Table 1 Baseline characteristics of participants.

\begin{tabular}{|c|c|c|}
\hline Measure & $\begin{array}{l}\text { Initially recruited cohort } \\
\qquad(n=245)\end{array}$ & $\begin{array}{l}\text { Finished exercise program } \\
\qquad(n=168)\end{array}$ \\
\hline Age (yrs) & $78.7 \pm 6.4$ & $78.3 \pm 6.3$ \\
\hline Gender (n, \% women) & $195,80.0 \%$ & $131,78.0 \%$ \\
\hline Mass (kg) & $75.9 \pm 18.4$ & $75.5 \pm 17.3$ \\
\hline $\mathrm{BMI}\left(\mathrm{kg} / \mathrm{m}^{2}\right)$ & $29.4 \pm 6.8$ & $29.6 \pm 6.6$ \\
\hline Medications (n) & $5.2 \pm 3.2$ & $5.1 \pm 3.1$ \\
\hline Morbidities (n) & $5.0 \pm 2.8$ & $4.8 \pm 2.6$ \\
\hline$A B C$ & $60.7 \pm 26.0$ & $65.5 \pm 24.3$ \\
\hline SPPB & $8.0 \pm 2.8$ & $8.6 \pm 2.5$ \\
\hline SPPB Balance (s) & $26.0 \pm 5.4$ & $26.8 \pm 5.1$ \\
\hline GDS & $3.1 \pm 2.4$ & $3.0 \pm 2.5$ \\
\hline GAI & $4.0 \pm 4.7$ & $3.9 \pm 4.6$ \\
\hline SARC-F ( $n, \%$ probable sarcopenic) & $85,34.7 \%$ & $34,20.2 \%$ \\
\hline EQ-5D-3L & $0.78 \pm 0.15$ & $0.79 \pm 0.14$ \\
\hline
\end{tabular}

2 All continuous data are presented as mean \pm standard deviation. All categorical data are presented as

3 the absolute number followed by the percentage. ABC: Activity-specific Balance Confidence

4 questionnaire. SPPB: Short Physical performance Battery. GDS: Geriatric Depression Scale. GAI: Geriatric

5 Anxiety Index. EQ-5D-3L: EuorQoL 5D 3L questionnaire. 


\section{Table 2 (on next page)}

Associations between SARC-F sum score or individual item response and objective measures of muscle function.

r: Pearson correlation. RR: Relative risk ratio moving from positive to negative SARC-F item response for a one standard deviation increase in objective measure. $*: p<0.05, * *: p<0.01$, $* * *: p<0.001$. 
1 Table 2. Associations between SARC-F sum score or individual item response and objective measures of

2 physical performance (95\% Confidence Intervals).

\begin{tabular}{lcccc}
\hline & $\begin{array}{l}\text { Isometric knee } \\
\text { extension strength }\end{array}$ & Handgrip strength & Walking speed & Chair-stand test \\
& & & & \\
\hline SARC-F Total Score (r) & $-0.21(-0.33 \text { to }-0.08)^{\dagger}$ & $-0.11(-0.23$ to -0.02$)$ & $-0.62(-0.74 \text { to }-0.51)^{\ddagger}$ & $0.57(0.48-0.65)^{\ddagger}$ \\
SARC-F Slowness (RR) & $0.93(0.62-1.1)$ & $0.92(0.69-1.2)$ & $0.31(0.20-0.47)^{\ddagger}$ & $2.1(1.5-2.9)^{\ddagger}$ \\
SARC-F Walking (RR) & $0.73(0.49-1.1)$ & $0.89(0.62-1.3)$ & $0.27(0.17-0.42)^{\ddagger}$ & $2.5(1.8-3.5)^{\ddagger}$ \\
SARC-F Chair (RR) & $0.67(0.38-1.2)$ & $0.92(0.57-1.5)$ & $0.12(0.06-0.27)^{\ddagger}$ & $3.5(2.2-5.5)^{\ddagger}$ \\
SARC-F Stairs (RR) & $0.53(0.36-0.79)^{\dagger}$ & $0.78(0.55-1.1)$ & $0.09(0.05-0.18)^{\ddagger}$ & $4.9(3.1-7.8)^{\ddagger}$ \\
SARC-F Falls (RR) & $0.43(0.19-0.97)^{*}$ & $0.53(0.27-1.0)$ & $0.31(0.15-0.61)^{\ddagger}$ & $1.80(1.1-3.0)^{*}$
\end{tabular}

3 r: Pearson correlation. RR: Relative risk ratio moving from positive to negative SARC-F item response for a one standard

4 deviation increase in objective measure. *: $p<0.05,+: p<0.01, \ddagger: p<0.001$ 


\section{Table 3 (on next page)}

Summary of SARC-F item and muscle function outcomes as a result of participation in the PRBT intervention.

Positive: No difficulty/0 falls in last year; INT (Intermediate): Some difficulty/1-3 falls in last year; Negative: A lot of difficulty/ $\geq 4$ falls in last year; ${ }^{+}$Change: Item score decreased from pre- to post-intervention/sarcopenic to non-sarcopenic, No change: Item score remained the same pre to post-intervention; Negative change: Item score increased from pre- to postintervention/non-sarcopenic to sarcopenic. 
1 Table 3 Summary of SARC-F item and muscle function outcomes as a result of participation in the PRBT intervention.

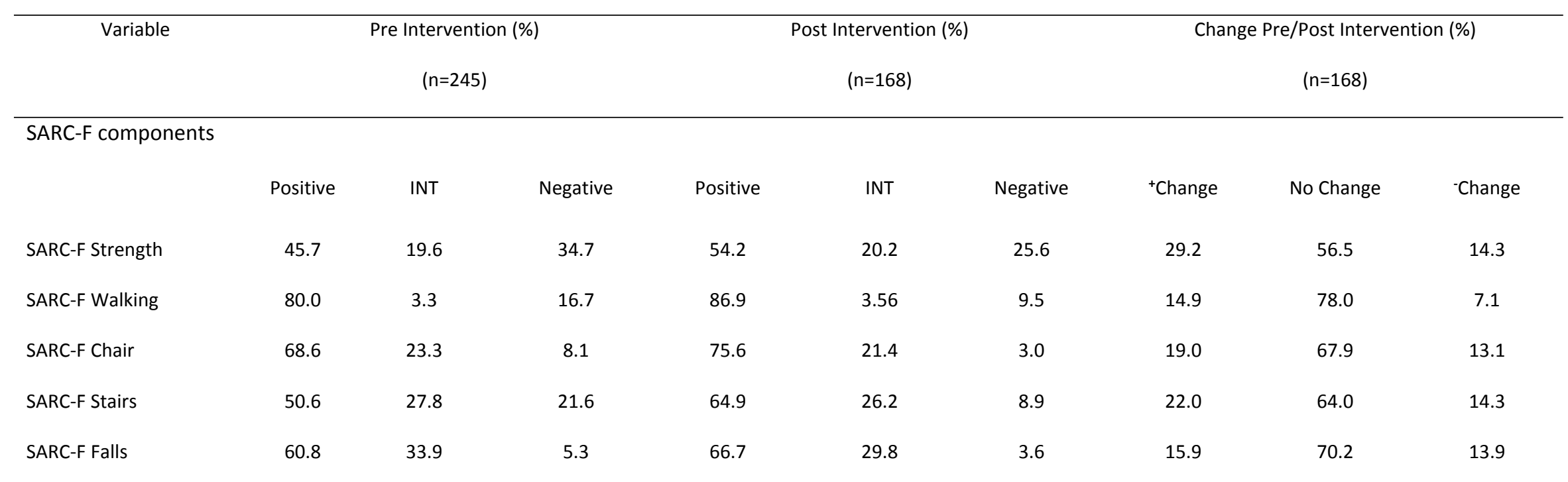

SARC-F Diagnosis

\begin{tabular}{|c|c|c|c|c|c|c|c|}
\hline & Sarcopenic & Non-Sarcopenic & Sarcopenic & Non-Sarcopenic & ${ }^{+}$Change & No Change & -Change \\
\hline Sarc-F Diagnosis & 34.7 & 65.3 & 20.2 & 79.8 & 13.7 & 79.2 & 7.1 \\
\hline Sarc-F Total Score & - & - & - & - & 44.1 & 31.0 & 24.9 \\
\hline
\end{tabular}

Training-related changes

Pre Intervention (Mean \pm SD)

$$
2.5 \pm(2.3)
$$

Post Intervention (Mean \pm SD)

$$
2.0 \pm(2.0)
$$

Change Pre/Post Intervention (Mean \pm SD)

$$
-0.5 \pm(2.0)
$$


strength $(\mathrm{kg})$

Handgrip Strength (kg)

2 Positive: No difficulty/0 falls in last year; INT (Intermediate): Some difficulty/1-3 falls in last year; Negative: A lot of difficulty/ $\geq 4$ falls in last year; ${ }^{+}$Change: Item

3 score decreased from pre- to post-intervention/sarcopenic to non-sarcopenic, No change: Item score remained the same pre to post-intervention; Negative

4 change: Item score increased from pre- to post-intervention/non-sarcopenic to sarcopenic. 


\section{Table 4 (on next page)}

Associations between changes in SARC-F sum score or individual item responses and change in objective measures of muscle function across intervention.

$A R^{2}$ : Adjusted $R^{2}$. RR: Relative risk ratio moving from positive to negative change in SARC-F item response for a one standard deviation increase in objective measure change. $*: p<0.05$, $* *: \mathrm{p}<0.01, * * *: \mathrm{p}<0.001$. 
1 Table 4. Associations between changes in SARC-F sum score or individual item responses and change in

2 objective measures of physical performance across intervention (95\% Confidence Intervals).

\begin{tabular}{lcccc}
\hline & Isometric strength & Handgrip Strength & Walking speed & Chair-stand test \\
\hline SARC-F Total Score (r) & $0.01(-0.14-0.16)$ & $-0.03(-0.18$ to -0.12$)$ & $-0.30(-0.43 \text { to }-0.15)^{\ddagger}$ & $0.33(0.18-0.45)^{\ddagger}$ \\
SARC-F Slowness (RR) & $0.76(0.46-1.3)$ & $0.95(0.57-1.6)$ & $0.63(0.37-1.1)$ & $2.2(1.2-4.1)^{\ddagger}$ \\
SARC-F Walking (RR) & $1.19(0.91-3.9)$ & $1.12(0.57-2.5)$ & $0.36(0.15-0.86)^{*}$ & $2.0(1.0-3.4)^{*}$ \\
SARC-F Chair (RR) & $1.32(0.76-2.3)$ & $0.82(0.46-1.5)$ & $0.47(0.24-0.92)^{*}$ & $2.1(1.2-3.8)$ \\
SARC-F Stairs (RR) & $0.83(0.48-1.4)$ & $0.57(0.32-1.0)$ & $0.40(0.21-0.76)^{\dagger}$ & $2.0(1.1-3.5)^{*}$ \\
SARC-F Falls (RR) & $0.92(0.51-1.7)$ & $1.13(0.73-2.4)$ & $0.94(0.52-1.7)$ & $1.11(0.60-2.0)$
\end{tabular}

3 r: Pearson correlation. RR: Relative risk ratio moving from positive to negative SARC-F item response for a one standard

4 deviation increase in objective measure. ${ }^{*}: p<0.05, \uparrow: p<0.01, \neq: p<0.001$.

5 